\title{
The Research and Design of Wearable Smart Blind Guidance
}

\author{
Rui Xue ${ }^{1, a_{*}}$,Shixian Zeng ${ }^{1, b}$ \\ ${ }^{1}$ Qingdao Huanghai College, China \\ axuerui126@126.com, ${ }^{\mathrm{b}} \mathrm{zs}$ 113@126.com
}

\section{Keywords: Blind Guidance; MSP430F1232; Data Acquisition System}

\begin{abstract}
In order to solve the deficiencies of the prior art. We present an intelligent wearable with guide blind shoes. It can detect the road surface and the convex surface on the ground of the obstacle position and distance accurately, and can help the blind to determine their specific position accurately, but also planning the travel route.
\end{abstract}

\section{Introduction}

At present, the patients with low vision and blind travelers face the difficulty at home or abroad, We are trying to explore a wide variety of facilities and portable systems guide blind, There has been developed electronic guide blind glasses successfully, radio guide blind cane, guide the blind satellite, infrared blind guidance, traffic light recognition systems, These systems or devices just solution difficult for a small area or blind travelers faced one or a few obstacles given, not to the blind travel to provide comprehensive help a whole ${ }^{[1][2][3]}$. We propose a wearable smart shoes guide the blind will lead the blind configuration in a shoe, the complete liberation of the user's hands ${ }^{[4][5]}$. The ultrasonic probe in different directions on the shoe, can be vertical and horizontal scanning probe was also more effective detection of obstacles ${ }^{[6][7]}$.

\section{System Design}

This guide includes a blind hardware circuit modules, communication modules, and voice broadcast module, Figure 1 is a block diagram of the wearable smart guide is blind. Hardware circuit module comprises a hardware circuit module symmetrical left and right hardware circuit modules, the left and right hardware circuit modules are mounted on the bottom of the left and right blind shoe, comprising a sensor, and a microprocessor, a sensor detects an obstacle on the road information ${ }^{[8]}$, and the detection of the transmission of information to the microprocessor processing, microprocessor control commands issued by the communication module, voice broadcast module to broadcast blind control commands $^{[9]}$. Wearable Smart blind guidance on the communication module includes a Bluetooth module and GPS module, Bluetooth module and GPS module are connected to different ports on the microprocessor.

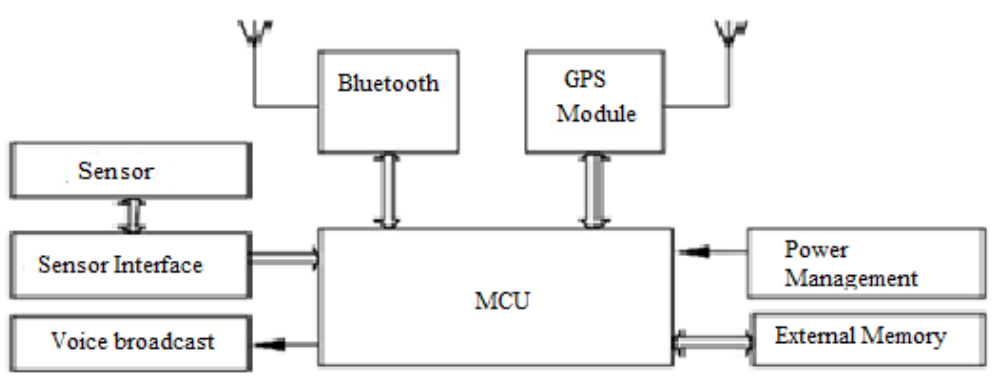

Figure 1 Block diagram of wearable intelligent guide blind control

Hardware Circuit. Hardware circuit module further comprises a left motor drive circuit, a Bluetooth transceiver circuit module and an ultrasonic transducer, a microprocessor connected to the motor drive circuit, the motor drive circuit connected to the motor, before the motor, and the left 
motor, an ultrasonic sensor transceiver circuit connected to the microprocessor, ultrasonic sensor transceiver circuit is connected to the sensor, the sensor and the front left sensor. the right hardware circuit module further includes a motor drive circuit, a Bluetooth module and an ultrasonic sensor transceiver circuits, microprocessors connecting the motor drive circuit, the motor drive circuit is connected to the motor, the motor and the front right motor, an ultrasonic sensor transceiver circuit connected to the microprocessor, an ultrasonic sensor transceiver circuit connected to the sensor, the sensor and the front right sensor, Figure 2 is a schematic block diagram of the right hardware circuit.

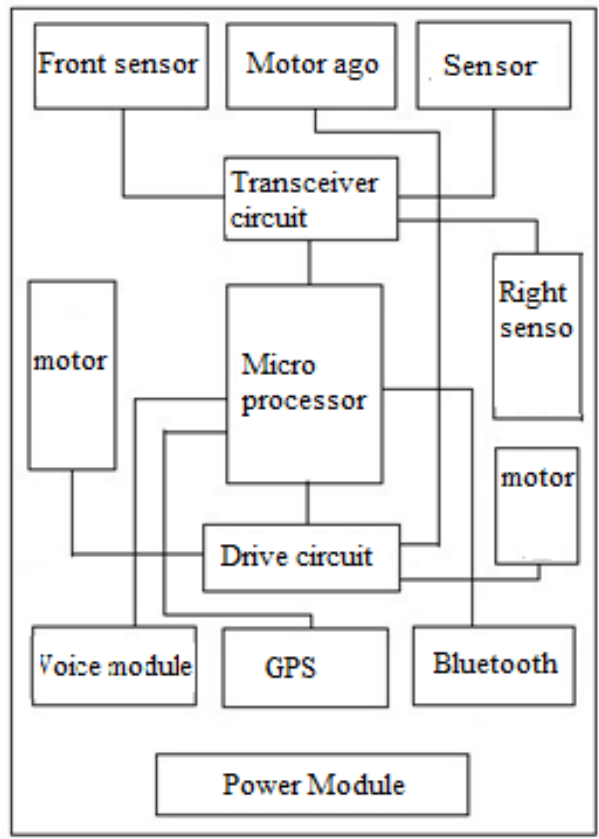

Figure 2 Right module hardware circuit schematic

Processor. We chose MSP430F1232 as microprocessor, it has 64 KB of FLASH program memory and $64 \mathrm{MB}$ of RAM with MSP430F1232 microcontroller inside ${ }^{[9][10]}$, there is a dual universal asynchronous serial ports in the internal microcontroller.

Voice Module. We will use the Bluetooth headset as voice broadcast module, which communicate via a Bluetooth module and a microprocessor. It contains speech conversion circuit voice broadcast module, we use voice chip ISD4000, ISD4000 voice chip embedded in the crystal oscillator system ${ }^{[7]}$, anti-aliasing filtering system, filtering system, automatic squelch, audio power amplifier system, it just few external member could constitute a complete system of voice recording systems. In order to improve the ability to drive the speaker system, using the LM386 power amplifier system. Figure 3 is a schematic diagram of a voice conversion circuit.

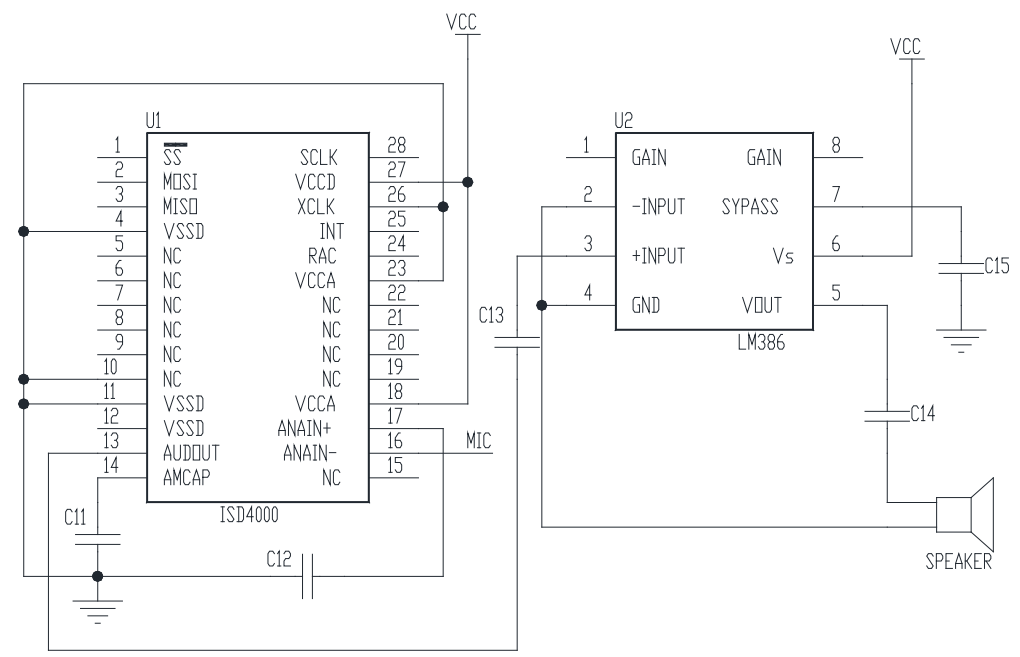

Figure 3 Speech conversion circuit schematic 
Ultrasonic module. It ultrasonic transmitting circuit and the receiving circuit of the ultrasonic transceiver circuit by the ultrasonic sensor, ultrasonic transmitter circuit comprises an ultrasonic transducer, Figure 4 is a schematic view of an ultrasonic transmitter circuit. Ultrasonic receiver circuit includes an output detector receiver chip, the microprocessor square wave emitted by the ultrasonic transducer, the echo received by the detector receiver chip, the microprocessor note of the ultrasonic echo time, calculates the distance between obstacles. We use P1.0 port output ultrasonic transducer required $40 \mathrm{kHz}$ square wave signal using external interrupt port monitor ultrasonic receiver circuit output return signal, Figure 5 is a schematic view of an ultrasonic receiver circuit.

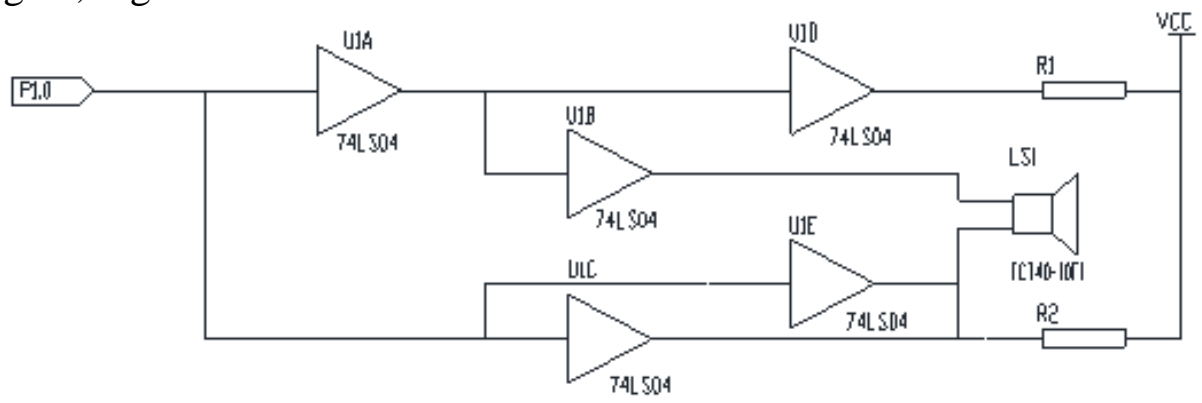

Figure 4 Ultrasonic transmitter circuit schematic

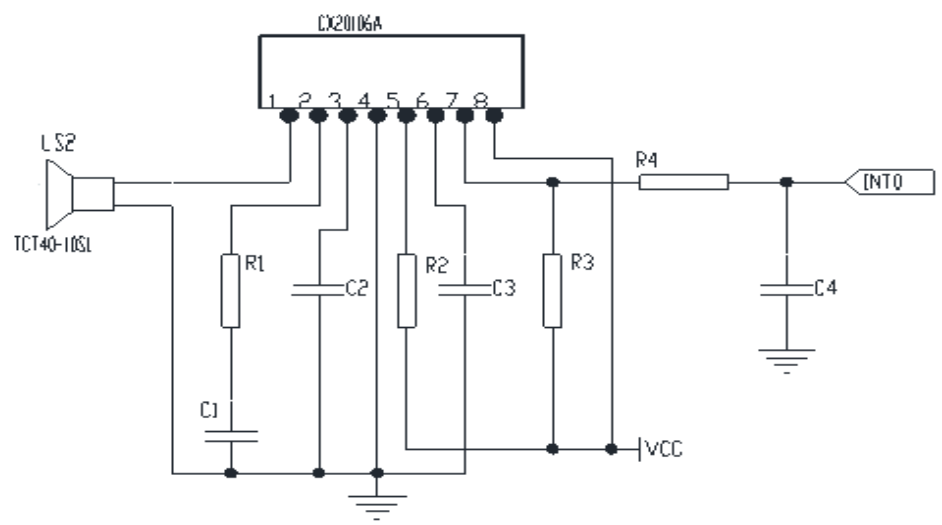

Figure 5 Ultrasonic receiver circuit schematic

Motor Circuit. Pulse motor drive circuit input terminal and the output terminal of the microprocessor is connected, through the transistor bridge circuit composed of complementary motor drive circuit and the output of the motor is connected, through the PWM pulse width control method to control the motor speed, advantage of the bridge is simple in principle, easy to control, with a load capacity of at MSP430F1232 with by PWM pulse width control method ${ }^{[8]}$, the realization of the motor speed control. Figure 6 is a schematic view of the motor drive circuit.

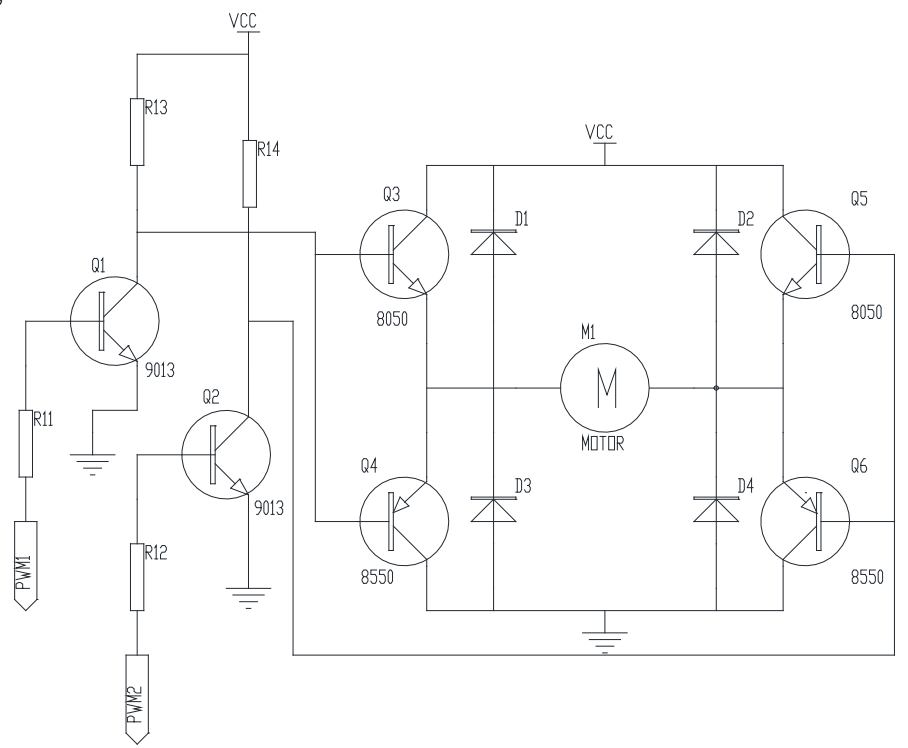

Figure 6 The motor drive circuit schematic 
Power Module. Boosting power supply circuit is a circuit configured LM2577, LM2577 is a typical boost switching power regulator integrated, P1 input voltage range (3.5V-40V), P2 output switching power supply up to 3A, the output voltage $12 \mathrm{~V}$ and $15 \mathrm{~V}$. LM2577 can be easily implemented using the boost conversion system, forward converter system conversion system control. DC-DC Boost module LM2577 chip composed of switching power supply ${ }^{[9]}$, to ensure that the output current is large, and the booster circuit module lithium battery $3.7 \mathrm{~V}$ raised to $5 \mathrm{~V}$, so that you can give power intelligent wearable device guide the blind. Figure 7 is a schematic block diagram of the power supply circuit.

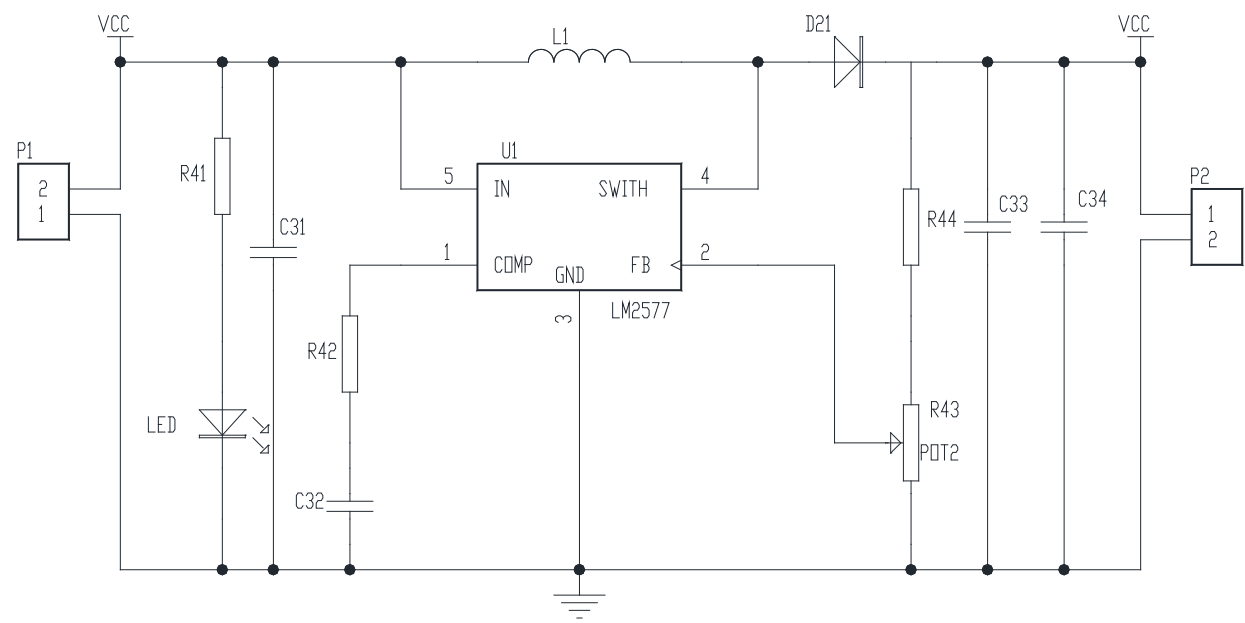

Figure 7 The power supply circuit schematic

\section{Operation and Summary}

When the command is issued destination, the system through GPS positioning module and choose the best route. Blind in the process of moving, real-time ultrasonic transmitter circuit transmitting ultrasonic signals, ultrasonic receiver circuit receives echo, echo received by the detector chip receiving ultrasonic echo time a note is microprocessor calculates the distance between obstacles. When the distance of the warning area, through the motor drive circuit drives the corresponding position of the motor , through the Bluetooth module and headset communications, voice prompts the user to reach the destination, but also commissioning a vibrate mode.

\section{References}

[1] R. Palit,S. Saha,J. Sethi,T. Trivedi,S. Sharma,B.S. Naidu,S. Jadhav,R. Donthi,P.B. Chavan,H. Tan,W. Hennig. A high speed digital data acquisition system for the Indian National Gamma Array at Tata Institute of Fundamental Research[J]. Nuclear Inst. and Methods in Physics Research, A,2012,680-691.

[2] Ammar Mahjoubi,Ridha Fethi Mechlouch,Ammar Ben Brahim. Data Acquisition System for Photovoltaic Water Pumping System in the Desert of Tunisia[J]. Procedia Engineering,2012,33-45.

[3] Jucá, Sandro César Silveira,Pereira, Renata Imaculada Soares,de Carvalho, Paulo Cesar Marques. WiFi Data Acquisition System Applied to a Photovoltaic Powered Water Pumping Plant[J]. Sensors \& Transducers,2015,1852-1861.

[4] A.Yu. Isupov,V.A. Krasnov,V.P. Ladygin,S.M. Piyadin,S.G. Reznikov. The Nuclotron internal target control and data acquisition system[J]. Nuclear Inst. and Methods in Physics Research, A,2013,698-710.

[5] Rachana Rajpal,Jigneshkumar Patel,Praveena Kumari,Vipul Panchal,P.K. Chattopadhyay,Harshad Pujara,Y.C. Saxena. Embedded data acquisition system with MDSPlus[J]. Fusion Engineering and Design,2012,8712-8720. 
[6] Song Qiang,Lv Chenguang. Data Acquisition System for Electric Vehicle's Driving Motor Test Bench Based on VC++[J]. Physics Procedia,2012,33-40.

[7] Yazhou Yuan,Qimin Xu,Xinping Guan,Zhixin Liu. Industrial high-speed wireless synchronous data acquisition system with real-time data compression[J]. Measurement,2013,469-478.

[8] SeungWoo Lee,So Jeong Nam,Jai-Kyung Lee. Real-time data acquisition system and HMI for MES[J]. Journal of Mechanical Science and Technology,2012,268-279.

[9] Pieralisi Marco,Petrini Valerio,Di Mattia Valentina,Manfredi Giovanni,De Leo Alfredo,Scalise Lorenzo,Russo Paola,Cerri Graziano. Design and Realization of an Electromagnetic Guiding System for Blind Running Athletes.[J]. Sensors (Basel, Switzerland),2015,157-167.

[10] Frank John Lane,Margaret Huyck,Philip Troyk,Kenneth Schug. Responses of potential users to the intracortical visual prosthesis: final themes from the analysis of focus group data[J]. Disability and Rehabilitation: Assistive Technology,2012,74-86. 